\title{
Qualidade de vida em portadores de glaucoma: comparação entre pacientes do sistema público de saúde e clínica privada
}

\author{
Quality of life in glaucoma patients: comparison between \\ public health system and private practice
}

Dalmo Pina Pinheiro', Maria Luiza Garcia Rosa², Luis Guillermo Coca Velarde², João Paulo Lomelino ${ }^{4}$, Paolo Esteves Rabelo Knopp ${ }^{5}$, Marcelo Palis Ventura ${ }^{6}$

\section{ResUMO}

Objetivo: Avaliar a qualidade de vida de portadores de glaucoma, comparando pacientes do sistema público de saúde com pacientes atendidos no sistema particular, através do Questionário de Função Visual de 25 itens do National Eye Institute (NEI-VFQ). Métodos: Realizou-se um estudo transversal, desenvolvido mediante a aplicação do questionário NEI-VFQ, em 83 pacientes glaucomatosos não consecutivos em diferentes estágios da doença, sendo 51 do Hospital Universitário Antonio Pedro (instituição pública), e 32 pacientes de clínica particular (instituição privada). Resultados: Houve diferença estatística significativa entre os grupos nos subdomínios referentes à "saúde geral", "visão geral", "dor ocular", "atividades para perto", "atividades para longe", "saúde mental" e "atividades de vida diária". Não foi encontrada diferença significativa nos subdomínios referentes a "aspectos sociais", "dependência", "capacidade para dirigir", "visão de cores" e "visão periférica". O escore geral demonstrou diferença estatística significativa entre os grupos, com média de 73,13 para instituição pública e 86,07 para instituição privada. Conclusão: O impacto do glaucoma na qualidade de vida foi maior nos pacientes de instituição pública, quando comparados com aqueles de instituição privada através do questionário NEI-VFQ.

Descritores: Qualidade de vida; Glaucoma; Saúde pública; Questionários

\footnotetext{
'Mestre em Ciências Médicas da Universidade Federal Fluminense - UFF - Niterói (RJ) - Brasil;

${ }^{2}$ Professora associada do Departamento de Epidemiologia da Universidade Federal Fluminense - UFF - Niterói (RJ), Brasil; ${ }^{3}$ D.Sc. Professor associado do Departamento de Estatística da Universidade Federal Fluminense - UFF - Niterói (RJ), Brasil; ${ }^{4}$ Residente do terceiro ano do Serviço de Oftalmologia da Universidade Federal Fluminense - UFF - Niterói (RJ), Brasil; ${ }^{5}$ Acadêmico do quinto ano do curso de Medicina da Universidade Federal Fluminense - UFF - Niterói (RJ), Brasil;

${ }^{6}$ Professor associado de Oftalmologia, Chefe do Setor de Glaucoma - Universidade Federal Fluminense - UFF - Niterói (RJ), Brasil.

Trabalho realizado no Setor de Glaucoma do Serviço de Oftalmologia da Universidade Federal Fluminense - UFF - Niterói (RJ), Brasil.
} 


\section{INTRODUÇÃO}

$\mathbf{S}$ egundo a Organização Mundial de Saúde (OMS), qualidade de vida é definida como "a percepção do indivíduo de sua posição na vida no contexto da cultura e sistema de valores nos quais ele vive e em relação aos seus objetivos, expectativas, padrões e preocupações" ${ }^{(1)}$. Em oftalmologia muitos questionários relacionados à função visual têm sido usados no intuito de avaliar o impacto de patologias ou intervenções na qualidade de vida dos pacientes. Desses questionários, alguns apresentam aspecto mais específico, sendo usados somente para determinadas patologias não podendo, desta forma, serem comparados diretamente entre doenças ${ }^{(2,3)}$. Para diferentes condições oftalmológicas foi desenvolvido o National Eye Institute 25-Item Visual Function Questionnaire (NEI-VFQ). Este questionário, que tem sido largamente utilizado, foi elaborado inicialmente com 51 itens e posteriormente reduzido e readaptado para sua versão de 25 itens, com a opção de acréscimo de 14 questões ${ }^{(4)}$.

O impacto das doenças na qualidade de vida é um conceito bem definido, já tendo sido observado em diferentes populações ${ }^{(5-7)}$. Diversos trabalhos têm fornecido informações relacionadas à influência negativa do glaucoma na qualidade de vida dos pacientes, inclusive com estudos realizados na população brasileira ${ }^{(8)}$.

O sistema de saúde brasileiro apresenta dois grupos distintos bem definidos, compostos de pacientes usuários do sistema público e aqueles usuários do sistema privado, nos quais as condições sócioeconômicas e culturais são extremamente diferentes. Portanto, a análise do impacto da qualidade de vida em grupos tão distintos pode fornecer informações importantes para ações diferenciadas dos gestores de saúde pública.

O objetivo deste estudo é avaliar a qualidade de vida de pacientes portadores de glaucoma, comparando pacientes do sistema público de saúde atendidos no Hospital Universitário Antonio Pedro, e pacientes de clínica particular, através do questionário NEI-VFQ.

\section{Métodos}

Após aprovação pelo Comitê de Ética e Pesquisa, realizou-se um estudo transversal, desenvolvido mediante a aplicação do questionário NEI-VFQ, em 51 pacientes glaucomatosos não consecutivos em diferentes estágios da doença, do Hospital Universitário Antonio Pedro (instituição pública), e em 32 pacientes de clínica particular (instituição privada), possuidores de planos de saúde ou particulares, no período de agosto de 2008 a março de 2009.

O NEI-VFQ é um instrumento de 25 itens com mais 14 questões opcionais, que foi desenvolvido para medir a função visual e o impacto na qualidade de vida para uma variedade de condições oculares ${ }^{(9)}$. Suas 25 questões são agrupadas em 12 subdomínios com uma ou mais questões em cada subdomínio, compreendendo: saúde geral, visão geral, dor ocular, atividades para perto, avidades para longe, aspectos sociais, saúde mental, atividades da vida diária, dependência, capacidade para dirigir, visão de cores e visão periférica. Para cada questão há cinco possibilidades de resposta, sendo que para cada uma obtém-se uma pontuação que varia de 0 a 100 $(0,25,50,75$ e 100 pontos, de acordo com a resposta). A pontuação final obtida é então dividida pelo número de questões, obtendo-se um escore geral para cada paciente, cujo valor mínimo é zero e o valor máximo é 100 . Quanto maior o escore alcançado, melhor a qualidade de vida, lembrando que no escore geral não se inclui o subdomínio 'saúde geral'.

No intuito de melhor avaliar algum subdomínio específico foram elaboradas, pelo próprio National Eye Institute, 14 questões adicionais que, por sugestão dos autores, devem ser aplicadas integralmente por subdomínio que se pretende melhor avaliar. Essa estratégia visa aumentar a comparabilidade dos resultados entre diferentes estudos, sendo assim também incluída neste estudo.

Os critérios de inclusão foram: idade mínima de 40 anos, diagnóstico de glaucoma primário de ângulo aberto há no mínimo um ano, possuir alteração glaucomatosa em campo visual em pelo menos um olho e estar em acompanhamento clínico no respectivo local. Foi considerado critério de exclusão a história de cirurgia ocular ou tratamento com laser nos três meses precedentes à aplicação do questionário. Termo de consentimento informado foi obtido após explicação ao paciente sobre a natureza do estudo. $\mathrm{O}$ tamanho da amostra foi calculado para que houvesse significância estatística de $5 \%$ com $95 \%$ de poder de teste.

Os questionários foram administrados via entrevista por dois médicos e um acadêmico de medicina, treinados e orientados pelo autor previamente, com voluntários não incluídos no estudo. Os pacientes eram recrutados espontaneamente na ordem natural de chegada em consulta e entrevistados em sala separada do local do exame em ambiente tranquilo.

Foram coletados os dados demográficos e clínicos de todos os pacientes incluídos no estudo.

Para análise da normalidade dos dados foi utiliza- 
Tabela 1

Dados demográficos e clínicos relevantes por grupo

\begin{tabular}{lllc}
\hline & Instituição pública & Instituição privada & Valor p \\
\hline Idade (média) & 63,6 & 67,6 & 0,134 \\
Sexo & Masc: $12(23,53 \%)$ & Masc: $13(40,63 \%)$ & 0,159 \\
& Fem: $39(76,47 \%)$ & Fem: $19(59,37 \%)$ & $<0,0001^{*}$ \\
Escolaridade (anos) & 5,06 & 10,69 & $<0,0001^{*}$ \\
Renda familiar média (reais) & 1229,18 & 4805,35 & 0,1812 \\
Número médio de pessoas por casa & 2,86 & 2,56 & 0,1752 \\
Campo visual (MD, dB) & OD: $-8,78$ & OD: $-9,80$ & 0,1534 \\
& OE: $-7,21$ & OE: $-9,63$ & 0,390 \\
Acuidade visual(LogMAR) & & & 0,425 \\
OD: 0,16 & OD: 0,19 & 0,819 \\
\hline
\end{tabular}

$(*)=$ diferença significativa; Masc $=$ pacientes do sexo masculino; $F e m=$ pacientes do sexo feminino; $\mathrm{MD}=$ mean deviation; $\mathrm{dB}=$ decibéis

Tabela 2

Análise comparativa do escore geral e por subdomínios entre pacientes de instituição pública e pacientes de instituição privada

\begin{tabular}{|c|c|c|c|}
\hline Escore geral e subdomínios & Instituição pública & Instituição privada & $\begin{array}{l}\text { Teste de Wilcoxon } \\
\text { (valor p) }\end{array}$ \\
\hline Escore geral & 73,13 & 86,07 & $0,0002 *$ \\
\hline Saúde geral & 82,75 & 62,97 & $0,0184 *$ \\
\hline Visão geral & 62,20 & 75,62 & $0,0382 *$ \\
\hline Dor ocular & 46,32 & 85,55 & $0,0001 *$ \\
\hline Atividades para perto & 72,46 & 90,92 & $0,0002 *$ \\
\hline Atividades para longe & 69,95 & 80,46 & $0,0495^{*}$ \\
\hline Aspectos sociais & 89,79 & 96,35 & $0,071 \mathrm{NS}$ \\
\hline Saúde mental & 62,94 & 80,15 & $0,0023^{*}$ \\
\hline Atividades de vida diária & 78,06 & 93,16 & $0,0013^{*}$ \\
\hline Dependência & 82,48 & 91,21 & $0,1131 \mathrm{NS}$ \\
\hline Capacidade de dirigir & 47,50 & 60,94 & $0,7478 \mathrm{NS}$ \\
\hline Visão de cores & 94,60 & 99,22 & $0,0726 \mathrm{NS}$ \\
\hline Visão periférica & 73,53 & 79,69 & $0,2771 \mathrm{NS}$ \\
\hline
\end{tabular}

NS = não significativa; $(*)=$ significativa

do o teste de Kolmogorov-Smirnov. No caso das variáveis que apresentaram distribuição não normal, foram utilizados os testes de Wilcoxon e Kruskal-Wallis, o primeiro para analisar as variáveis individualmente entre os grupos e, o segundo, quando houve necessidade de avaliação para comparações de vários grupos. Para a análise das correlações não paramétricas utilizou-se o teste de Spearman, sendo consideradas diretas fracas quando " $p$ " estivesse entre 0 e 0,3 ; diretas moderadas quando "p" estivesse entre 0,3 e 0,5 , e fortes quando maior que 0,5 (10). Significância estatística foi considerada quando o valor de $\mathrm{p}$ fosse menor que 0,05 . A análise estatística foi realizada utilizando-se o programa S-Plus 8.0 (S-PLUS ${ }^{\circledR}$ 8 for Windows, Insightful Corporation, Seatle.WA).

\section{Resultados}

O estudo compreendeu um total de 83 pacientes, sendo 51 do sistema público de saúde e 32 da clínica privada. Dados relativos aos achados clínicos oftalmológicos e demográficos constam na tabela 1. 
Houve diferença estatística significativa entre os grupos, quando avaliada "renda familiar" e "escolaridade" (valor $\mathrm{p}<0,0001$ ). Foi avaliada a correlação destas variáveis ao escore geral de qualidade de vida que se mostrou não significativa tanto no grupo instituição pública (renda: $\mathrm{p}=0,2296$, escolaridade: $\mathrm{p}=0,1693$ ) quanto no grupo instituição privada (renda: $p=0,9931$, escolaridade: $\mathrm{p}=0,4472$ ). Altas ametropias, visão monocular e retinopatia diabética não permitiram análise para inferência estatística, devido ao número muito pequeno ou ausente de pacientes que apresentavam essas condições.

Os escores gerais médios por grupo foram: instituição pública de 73,13 \pm 18 ,99 e instituição privada de $86,07 \pm 12,42$, sendo observada diferença estatística significativa com $p$-valor de 0,0002 . Também foi observada diferença estatística significativa nos subdomínios "saúde geral", "visão geral", "dor ocular", "atividades para perto", "atividades para longe", "saúde mental" e "atividades de vida diária”(Tabela 2).

\section{DiscussÃo}

Segundo a Organização Mundial de Saúde, saúde é "um estado de completo bem-estar físico, mental e social, e não apenas a ausência de doença”, sendo seu conceito o norteador dos profissionais dessa área. Deste modo, a importância da avaliação da qualidade de vida nos pacientes portadores de glaucoma é assunto atual e de grande importância, principalmente pelo impacto resultante da evolução final da doença. Dados mostram que os gastos com a doença aumentam substancialmente, conforme a gravidade da patologia, gerando um impacto importante nos gastos dos sistemas públicos e privados de saúde ${ }^{(11)}$.

A idéia inicial deste estudo partiu da dúvida se pacientes com glaucoma de hospitais públicos teriam um impacto maior na qualidade de vida quando comparados com aqueles de intituições privadas. No Brasil existe uma acentuada diferença sócioeconômico/cultural entre esses grupos, sendo que a maioria da população de baixa renda é dependente do sistema público de saúde. Embora trabalhos anteriores tenham avaliado de diversas formas a qualidade de vida de pacientes portadores de glaucoma, não encontramos dados literários comparativos diretos a esse respeito e com esse fim.

A confiabilidade e validade do NEI-VFQ foram demonstradas em sua elaboração, sendo aplicado para pacientes com diferentes doenças oftamológicas ${ }^{(12)}$. No Brasil este questionário vem sendo utilizado desde 2001, tendo a sua consistência interna e reprodutibilidade tes- te-reteste demonstradas, estando validado e disponível para a língua portuguesa ${ }^{(13-14)}$.

Dados da literatura sugerem que algumas condições podem interferir na qualidade de vida de portadores de glaucoma como a idade, campo visual, acuidade visual e o nível educacional ${ }^{(15-19)}$.

As possíveis variáveis de interferência apresentaram-se homogêneas nos grupos estudados, com exceção das variáveis renda e escolaridade, condições implícitas a cada grupo, não modificadas, devido à opção de amostragem por demanda aleatória. Esses dados parecem ser justificáveis, visto que no Brasil conhece-se literal e observacionalmente a existência de diferenças de classes sócioeconômico culturais embora esteja atualmente classificado como país com elevado índice de desenvolvimento humano ${ }^{(20,21)}$.

Devido a essa diferença, essas duas variáveis foram analisadas na busca de correlação ao escore geral de qualidade de vida, não sendo observada associação significativa em nenhum dos grupos. Desta forma não pudemos atribuir a nenhuma destas variáveis a diferença encontrada no escore geral.

Dados de revisão de literatura demonstraram escores diferentemente baixos em pacientes glaucomatosos nos subdomínios "atividades de vida diária”,"capacidade para dirigir", "visão periférica” e "dependência"; desses itens nossos grupos diferiram entre si somente em "atividades de vida diária"(22-23).

Parece haver uma tendência do paciente em avaliar de modo mais otimista sua qualidade de vida do que julgam os oftalmologistas ${ }^{(24)}$. Observamos neste estudo que a diferença na percepção do paciente quanto a sua qualidade de vida parece se relacionar consideravelmente ao seu contexto de inserção social, e faz-nos pensar em quão perto da patologia estamos e quão longe do paciente em seu contexto global. Repensar estratégias de tratamento e prevenção é o que os instrumentos de avaliação têm nos sugerido, lembrando que cabem tratamentos e cuidados também individualizados, especialmente a uma parcela da população que além de apresentar diferentes tipos de limitações sócioeconômico/ culturais, sofre um maior impacto pelo glaucoma na sua qualidade de vida.

\section{Abstract}

Purpose: To evaluate the quality of life of glaucoma patients comparing public health system, and private practice, through the questionnaire National Eye Institute 25-Item Visual Function Questionnaire (NEI-VFQ). 
Methods: A cross-sectional study, developed through the application of NEI-VFQ questionnaire in 83 nonconsecutive glaucoma patients at different stages of the disease, and 51 from public health system, and 32 patients from private clinic. Results: Statistically significant difference between groups was demonstrated in subdomains related to the "general health", "general vision", "ocular pain", "near activities", "distance activities", "mental health" and "role difficulties" no significant difference was demonstrated in "social functioning", "dependency", "driving", "color vision" and "peripheral vision". Regarding the overall score given by the questionnaire statistical difference was observed with an average of 73.13 in the public institutions and 86.07 in the private practice. Conclusion: The impact on quality of life was higher in patients from the public institution when compared to those of the private practice through the NEI-VFQ questionnaire.

Keywords: Quality of life; Glaucoma; Public health; Questionnaires

\section{ReFERÊNCIAS}

1. World Health Organization Quality of Life Group. Development of the WHOQOL: Rationale and Current Status. Int J Ment Health. 1994;23(1):24-56.

2. Spaeth G, Walt J, Keener J. Evaluation of quality of life for patients with glaucoma. Am J Ophthalmol. 2006;141(1 Suppl):S3-14.

3. Goldberg I, Clement CI, Chiang TH, Walt JG, Lee LJ, Graham S, Healey PR. Assessing quality of life in patients with glaucoma using the Glaucoma Quality of Life-15 (GQL-15) questionnaire. J Glaucoma. 2009;18(1):6-12.

4. Mangione CM, Lee PP, Gutierrez PR, Spritzer K, Berry S, Hays RD, National Eye Institute Visual Function Questionnaire Field Test Investigators. Development of the 25-item National Eye Institute visual function questionnaire. Arch Ophthalmol. 2001;119(7):1050-8.

5. Nordmann JP, Viala M, Sullivan K, Arnould B, Berdeaux G. Psychometric Validation of the National Eye Institute Visual Function Questionnaire - 25 (NEI VFQ-25) French version: in a population of patients treated for ocular hypertension and glaucoma. Pharmacoeconomics. 2004;22(3):197-206.

6. Illarionova AR Study of the quality of life in patients with glaucoma.Vestn Oftalmol. 2003;119(3):11-5.

7. Rossi GC, Milano G, Tinelli C. The Italian version of the 25-item National Eye Institute Visual Function Questionnaire: translation, validity, and reliability. J Glaucoma. 2003;12(3):213-20.

8. Cypel MC, Kasahara N, Atique D, Umbelino CC, Alcântara MP, Seixas FS, de Almeida GV, Mandia C Jr, Cohen R. Quality of life in patients with glaucoma who live in a developing country. Int Ophthalmol. 2004;25(5-6):267-72.

9. Mangione CM, Berry S, Spritzer K, Janz NK, Klein R, Owsley $\mathrm{C}$, et al. Identifiyng the content area for the 51-item National Eye Institute visual functional questionnarie: results focus groups with visually impaired persons. Arch Ophthalmol. 1998;116(2):227-33.
10. Altman DG. Practical Statistics for Medical Research. London: Chapman \& HAll; 1991.

11. Calissendorff BM. Costs of medical and surgical treatment of glaucoma. Acta Ophthalmol Scand. 2001;79(3):286-8

12. Mangione CM, Lee PP, Pitts J, Gutierrez P, Berry S, Hays RD, and the NEI-VFQ Field Test Investigators. Psychometric properties of the National Eye Institute visual function questionnaire (NEI-VFQ). Arch Ophthalmol. 1998;116(11):1496-504.

13. Belfort R. Avaliação de qualidade visual e de vida de pacientes portadores de ametropias [tese]. São Paulo: Universidade Federal de São Paulo; 2001.

14. Simão LM, Lana-Peixoto MA, Araújo CR, Moreira MA, Teixeira AL. The Brazilian version of the 25-Item National Eye Institute Visual Function Questionnaire: translation, reliability and validity. Arq Bras Oftalmol. 2008;71(4):540-6.

15. McKean-Cowdin R, Varma R, Wu J, Hays RD, Azen SP; Los Angeles Latino Eye Study Group. Severity of Visual Field Loss and Health-related Quality. Am J Ophthalmol. 2007;143(6):1013-23.

16. Magacho L, Lima FE, Nery AC, Sagawa A, Magacho B, Avila MP. Quality of life in glaucoma patients: regression analysis and correlation with possible modifiers. Ophthalmic Epidemiol. 2004;11(4):263-70.

17. Wren PA, Musch DC, Janz NK, Niziol LM, Guire KE, Gillespie BW; Group, CIGTS Study. Contrasting the use of 2 visionspecific quality of life questionnaires in subjects with openangle glaucoma. J Glaucoma. 2009;18(5):403-11.

18. Labiris G, Katsanos A, Fanariotis M, Zacharaki F, Chatzoulis D, Kozobolis VP. Vision-specific Quality of Life in Greek Glaucoma Patients. J Glaucoma. 2010;19(1)39-43.

19. Gupta V, Srinivasan G, Mei SS, Gazzard G, Sihota R, Kapoor KS. Utility values among glaucoma patients: an impact on the quality of life. Br J Ophthalmol. 2005;89(10):1241-4.

20. Programa das Nações Unidas para o Desenvolvimento. Relatório de Desenvolvimento Humano - Brasil 2005. São Paulo: PrimaPagina; 2005.

21. Programa das Nações Unidas para o Desenvolvimento. Relatório de Desenvolvimento Humano- 2007/2008. Coimbra: Edições Almedina AS; 2007.

22. Gutierrez P, Wilson MR, Johnson C, Gordon M, Cioffi GA, Ritch $\mathrm{R}$, et al. Influence of glaucomatous visual field loss on health-related quality of life. Arch Ophthalmol. 1997;115(6):777- 84.

23. Parrish RK 2nd, Gedde SJ, Scott IU, Feuer WJ, Schiffman JC, Mangione CM, et al. Visual function and quality of life among patients with glaucoma. Arch Ophthalmol. 1997;115(11):1447-55.

24. Stein JD. Disparities between ophthalmologists and their patients in estimating quality of life. Curr Opin Ophthalmol. 2004;15(3):238-43.

\section{Endereço para correspondência: Dalmo Pina Pinheiro}

Av. Oswaldo Cruz no 96 - Apto 1207 - Flamengo

CEP 22250-060 - Rio de Janeiro (RJ), Brasil

Tel: (21) 32874704 / (21) 82079333

dalmo77@yahoo.com.br 